\title{
CONTRIBUTION A L'ÉTUDE DE LA VALEUR PRATIQUE DES TABLES D'ALIMENTATION
}

\author{
par
}

\section{ANDRÉ M. LEROY}

Directeur du Centre ambulant de Recherches zootechniques de l'Office Régional du Nord.

(Fin.)

\section{EXPÉRIENGES DE BONNELLES}

(du mois de Janvier au mois de Mai 1932). CHOIX DES ANIMAUX D'EXPÉRIENGE

PREMIER GROUPE : VACHES A RENDEMENT MOYEN

\begin{tabular}{|c|c|c|c|c|c|c|c|}
\hline Numéro du sujet $\ldots \ldots \ldots \ldots$ & 32 & 33 & 29 & 31 & 30 & 34 & 2 \\
\hline Race & Holl. & Holl. & Holl. & Holl. & Holl. & Holl. & Holl. \\
\hline Age $\ldots \ldots \ldots \ldots \ldots . . . . .$. & 7 ans & 6 ans & 8 ans & 6 ans & 6 ans & 6 ans & 7 ans: \\
\hline Dernier vêlage $\ldots \ldots \ldots \ldots$ & $4 / 9-31$ & $1 / 10-31$ & $14 / 9 \cdot 31$ & $24 / 12-31$ & $8 / 12-31$ & $13 / 11-31$ & $4 / 4-31$ \\
\hline $\begin{array}{l}\text { Rendement à } 4 \% \text { au mo- } \\
\text { ment du maximum (kg.) }\end{array}$ & 909 & 180 & 907 & 238 & 17.7 & 201 & 23,6 \\
\hline Poids vif moyen $(\mathrm{kg}.) \quad \ldots$ & 651 & 645 & 636 & 637 & 625 & 596 & 616 \\
\hline
\end{tabular}

SECOND GROUPE : VACHES A RENDEMENT ÉLEVÉ

Numéro du sujet $. \ldots \ldots \ldots . \quad 28$

Race .............. Holl.

Age $\ldots \ldots \ldots \ldots \ldots \ldots$ ans

Dernier vêlage ........ 26/2-32

Rendement à $4 \%$ au moment du maximum (kg.)

Poids vif moyen (kg.) ....
27

Holl.

10 ans

$25 / 2-32$

\section{4,5}

650

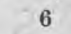

Holl.

8 ans

$27 / 2-32$
20

Holl.

6 ans

8/3-32

Holl.

7 ans

20/3-32:

17/3-32

24,9

606

VALEURS NUTRITIVES DES ALIMENTS UTILISÉS calculées au moyen d'analyses faites par le Laboratoire de la Société des Agriculteurs de France.

Bətteraves.... (Moyenne de 360 déterminations)

Foin de trèfle ............... ( 3 analyses)

Refus de foin................ (1 analyse)

Balles d'avoine ................ (3 analyses)

Cosses de fèves ............... (2 a nalyses)

Farine d'arachide déshuilée ........ (2 analyses)

Son de blé ................. (2 analyses)

Son d'arachide ............... (2 analyses)

Orge ................... (1 analyse)

Semoule de maïs ............. (2 analyses)

Tourteau de lin ................ (2 analyses)

Globazote ................. (2 analyses)

Concentré $\mathrm{N}^{0} 1$ (orge, son, arachide,

lin) $\ldots \ldots \ldots \ldots \ldots \ldots \ldots \ldots \ldots \ldots \ldots \ldots \ldots \ldots \ldots$ (1 analyse $)$

Concentré $\mathrm{N}^{\circ} 2$ (au son d'arachide) .. (1 analyse)
Quantité Album. digest. pour 1 U. F. par (en kilogramme kilogrammes) (en grammes)

$\begin{array}{rr}11,250 & 1 \\ 2,800 & 45 \\ 3,430 & 33,5 \\ 2,320 & 31 \\ 3,200 & 47 \\ 0,960 & 470 \\ 1,450 & 95 \\ 1,130 & 192 \\ 0,980 & 70 \\ 1,350 & 185 \\ 1,075 & 235 \\ 1,075 & 430\end{array}$

$1,100 \quad 152$.

$1,130 \quad 141$ 


\section{RÉSULTATS DU GONTROLE DE L'ALIMENTATION}

PÉriodè 1 (đu 15 au 31 Janvier 1932).

10 Ration de base :

Betteraves ........................ $39 \mathrm{~kg}$.

Balles ........................... $3 \mathrm{~kg}$.

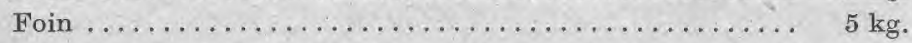

$2^{\circ}$ Composition du mélange concentré :

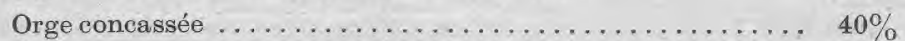

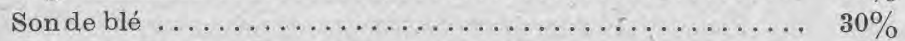

Tourteau d'arachide..................... $20 \%$

Tourteau de lin ........................ 10\%

Quantités de mélange concentré distribuées à chaque vache :

Premier groupe.

Numéro du sujet ...............

Quantité de mélange concentré (en

kilogrammes) ..............

Valeur fourragère totale de la ra-

tion, refus déduits (unités) ....

Quantités de mat. album. diges-

tibles (en grammes) $\ldots \ldots \ldots \ldots . \quad 1.328 \quad 994 \quad 996 \quad 1.462$

Second groupe : Non encore constitué.

Période 2 (du ler au 17 Février 1932).

10 Ration de base :

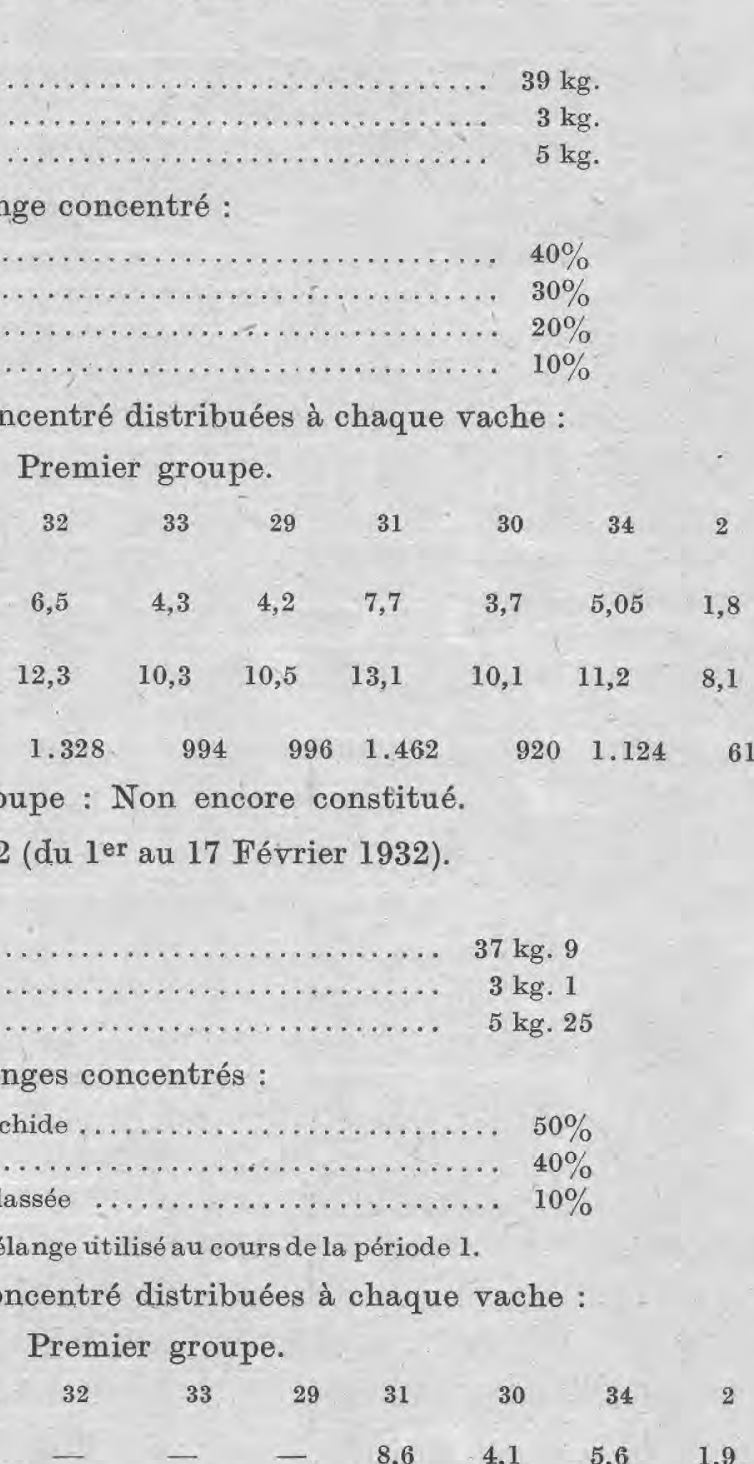

Betteraves $\ldots \ldots \ldots \ldots \ldots \ldots \ldots \ldots \ldots \ldots \ldots \ldots \ldots . \quad 37 \mathrm{~kg} .9$

Balles .......................... $3 \mathrm{~kg} .1$

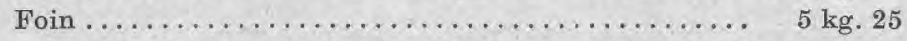

20 Composition des mélanges concentrés :

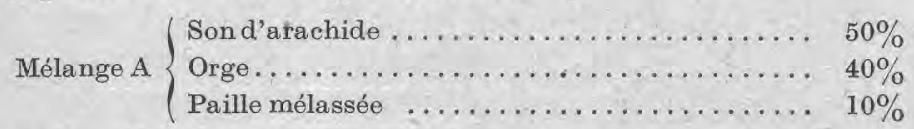

Mélange B : Voir le mélange uitilisé au eours de la période 1.

Quantités de mélange concentré distribuées à chaque vache :

Premier groupe.

Numéro du sujet

$32-33$

Quantité de mélange concentré (en kilogrammes)

$\begin{array}{lllllll}6,2 & - & - & 8,6 & 4,1 & 5,6 & 1,9\end{array}$

Valeur fourragère totale de la ration, refus déduits (unités) ......... Quantités de mat. album. digestibles (en grammes) ...........

$\begin{array}{lllllll}12,8 & 10,0 & 9,8 & 13,6 & 9,7 & 11,1 & 6,8\end{array}$

$\begin{array}{lllllll}1.313 & 1.005 & 980 & 1.553 & 933 & 1.156 & 632\end{array}$

Second groupe : Non encore constitué. 
Période 3 (du 18 au 23 Février 1932).

10 Ration de base :

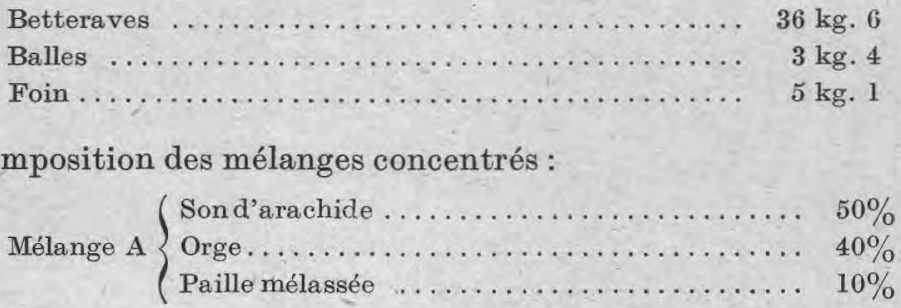

Mélange B : Voir le mélange utilisé pendant la période 1 .

Quantités de mélange concentré distribuées à chaque vache :

Premier groupe.

Numéro du sujet $\ldots \ldots \ldots \ldots \ldots \ldots \ldots . \quad 32 \quad 33 \quad 29 \quad 31 \quad 30 \quad 34 \quad 2$

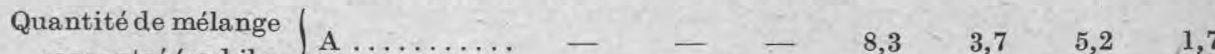

concentré (en kilo-
grammes) $\ldots \ldots \ldots$$\left\{\begin{array}{l}\text { A } \ldots \ldots \ldots \ldots \\ \text { B } \ldots \ldots \ldots \ldots\end{array}\right.$

Valeur fourragère totale de la ration,

refus déduits (unités) $\ldots \ldots \ldots \ldots \ldots \quad 11,4 \quad 9,9 \quad 9,3 \quad 13,5 \quad 9,1 \quad 10,8 \quad 7,7$

$\begin{array}{lllllllll}\text { Mat.album. digestibles (en grammes) } & 1.252 & 993 & 898 & 1.541 & 858 & 1.103 & 612\end{array}$

Second groupe: Non encore constitué.

Période 4 (du 26 Février au 1 er Mars).

10 Ration de base :

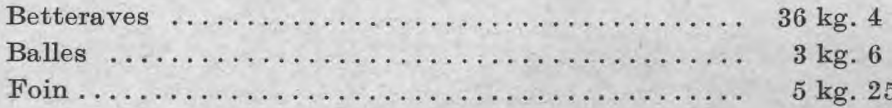

$2^{\circ}$ Composition des mélanges concentrés :

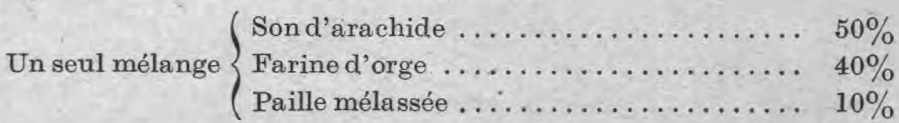

Quantités de mélange concentré distribuées à chaque vache :

Premier groupe.

Numéro du sujet............ $32 \quad 33 \quad 29 \quad 31 \quad 30 \quad 34 \quad$

Quantité de mélange concentré (en kilogrammes) ................

Valeur fourragère de la ration, refus déduits (unités) $\quad \ldots \ldots \ldots \ldots \ldots$.

$\begin{array}{lllllll}6,6 & 4,3 & 4,0 & 7,8 & 3,2 & 4,9 & 1,7\end{array}$

$\begin{array}{lllllll}12,1 & 10,1 & 9,8 & 13,0 & 8,9 & 10,3 & 7,8\end{array}$

$\begin{array}{llllllll}\text { Mat.album. digestibles (en grammes) } & 1.309 & 990 & 942 & 1.467 & 818 & 1.036 & 624\end{array}$

Second groupe : Non encore constitué. 
Période 5 (du 2 au 5 Mars).

$1^{\circ}$ Ration de base :

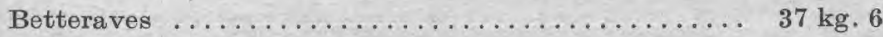

Balles .......................... $2 \mathrm{~kg} .4$

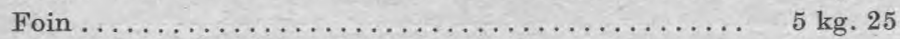

$2^{\circ}$ Composition du mélange concentré :

La même que celui de la période 4.

Quantités de mélange concentré distribuées à chaque vache :

\section{Premier groupe.}

Numéro du sujet $\ldots \ldots \ldots \ldots \ldots$

Quantité de mélange concentré (en kilogrammes) $\quad \ldots \ldots \ldots \ldots$.

Valeur fourragère de la ration, refus déduits (unités) ....... Mat. album, digestibles (en grammes) $\ldots \ldots \ldots \ldots \ldots \ldots \ldots$.

$\begin{array}{lccccccc}32 & 33 & 29 & 31 & 30 & 34 & 2 \\ 8,1 & 5,5 & 5,1 & 9,9 & 4,1 & 6,1 & 1,9 \\ 13,2 & 10,9 & 10,6 & 14,8 & 8,7 & 11,5 & 7,7 \\ 1.448 & 1.081 & 1.025 & 1.696 & 884 & 1.165 & 568 \\ \text { roupe : Non encore constitué. } & & & & \\ \text { ODE } 6 \text { (du } 6 \text { au } 10 \text { Mars). }\end{array}$

$1^{\circ}$ Ration de base :

Second groupe : Non encore constitué.

Période 6 (du 6 au 10 Mars).

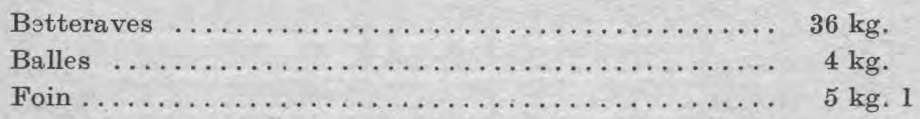

$2^{\circ}$ Composition du mélange concentré :

Le même que celui de la période 4.

Quantités de mélange concentré distribuées à chaque vache : Premier groupe.

\begin{tabular}{|c|c|c|c|c|c|c|c|}
\hline Numéro du sujets.............. & 32 & 33 & 29 & 31 & 30 & 34 & 2 \\
\hline (en kilogrammes) $\ldots \ldots \ldots \ldots$ & 8,1 & 5,5 & 5,1 & 9,9 & 4,1 & 6,1 & 1,9 \\
\hline $\begin{array}{l}\text { aleur fourragère de la ration, } \\
\text { refus déduits (unités) . . . . . . . } \\
\text { at. album. digestibles (en gram- }\end{array}$ & 13,2 & 10,9 & 10,5 & 14,7 & 8,6 & 11,4 & 7,7 \\
\hline mes) $\ldots \ldots \ldots \ldots \ldots \ldots \ldots$ & 1.546 & 1.181 & 1.126 & 1.792 & 984 & 1.266 & 668 \\
\hline
\end{tabular}

Période 7 (du 11 au 15 Mars).

10 Ration de base :

Betteraves ......................... 36 kg. 4

Balles d'avoine ....................... $3 \mathrm{~kg} .65$

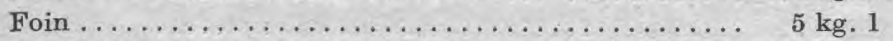


$2^{\circ}$ Composition du mélange concentré :

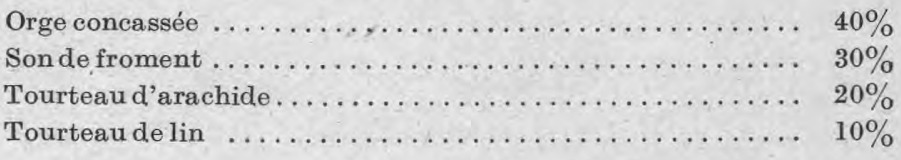

Quantités de mélange concentré distribuées à chaque vache :

Premier groupe.

Numéro du sujet $\ldots \ldots \ldots \ldots \ldots \ldots$.
Quantité de mélange concentré (en

kilogrammes) $\ldots \ldots \ldots \ldots \ldots \ldots \ldots \ldots \quad 5,6 \quad 3,7 \quad 3,4 \quad 8,0 \quad 2,6 \quad 4,1 \quad 1,5$

Valeur fourragère de la ration, refus

déduits (unités) $\ldots \ldots \ldots \ldots \ldots \ldots \ldots \quad 11,4 \quad 9,7 \quad 9,4 \quad 13,6 \quad 8,7 \quad 10,0 \quad 7,6$

$\begin{array}{llllllll}\text { Mat.album. digestibles (en grammes) . } & 1.223 & 940 & 889 & 1.591 & 772 & 1.001 & 602\end{array}$

Second groupe : Non encore constitué.

Période 8 (du 16 au 22 Mars).

$1^{\circ}$ Ration de base :

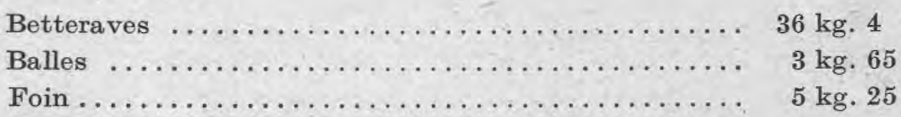

$2^{\circ}$ Composition du mélange concentré :

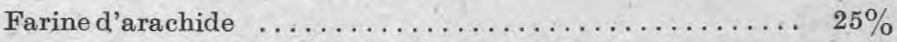

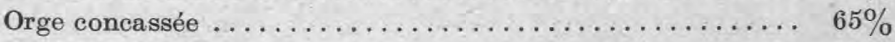

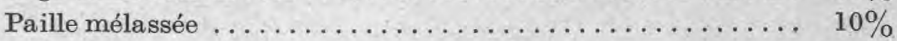

Quantités de mélange concentré distribuées à chaque vache :

Premier groupe.

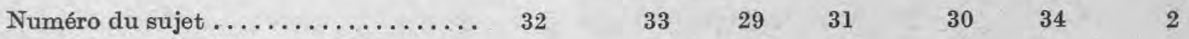

Quantité de mélange concentré (en

kilogrammes) $\ldots \ldots \ldots \ldots \ldots \ldots \ldots \ldots \quad 5,4 \quad 3,5 \quad 3,5 \quad 6,6 \quad 2,4 \quad 3,9 \quad 1,5$

Valeur fourragère de la ration, refus

déduits (unités) $\ldots \ldots \ldots \ldots \ldots \ldots \ldots \quad 11,6 \quad 9,8 \quad 9,9 \quad 12,9 \quad 8,8 \quad 10,3 \quad 7,9$

$\begin{array}{llllllll}\text { Mat. album. digestibles (en grammes). } & 1.240 & 941 & 958 & 1.446 & 770 & 1.012 & 621\end{array}$

Second groupe.

Numéro du sujet. ................... 28

Quantité de mélange concentré (en kilogrammes) ..... $\quad 7,4$

Valeur fourragère de la ration, refus déduits (unités) $\ldots \quad 13,6$

27

6

Mat.album.digestibles (en grammes)

1.563

7,6

6,0

13,8

12,3

Période 9 (du 23 au 26 Mars).

$1^{0}$ Ration de base :

Betteraves

Balles

Foin 
$2^{\circ}$ Composition du mélange concentré :

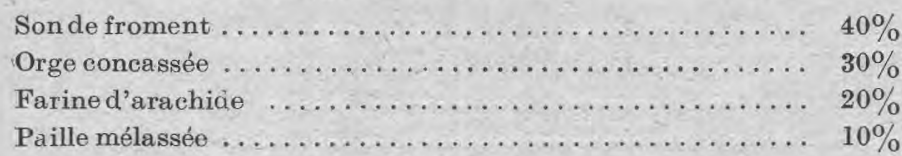

Quantités de mélange concentré distribuées à chaque vache :

\section{Premier groupe.}

Numéro du sujet .................
Quantité de málange concentré (en

kilogrammes) ...............

$\begin{array}{lllllll}6,1 & 4,0 & 4,1 & 7,6 & 2,8 & 4,4 & 1,5\end{array}$

Valeur fourragère de la ration, refus déduits (unités) ............. Mat. album. digestibles (en grammes)

$\begin{array}{llllllll}11,7 & 9,9 & 10,0 & 12,9 & 8,9 & 10,3 & 7,8 \\ 1.308 & 983 & 1.004 & 1.528 & 803 & 1.051 & 600\end{array}$

Second groupe.

Numéro du sujet. . . . . . . . . . . . . . . . 28

Quantité de mélange concentré (en kilogrammes) ...... 8,4

Valeur fourragère de la ration, refus déduits (unités) ...

13,7

6

Mat.album. digestibles (en kilogrammes)

1.651

8,6

6,8

13,8

12,3

1.698

1.417

Pértode 10 (du 27 au 31 Mars).

$1^{0}$ Ration de base :

Botteraves ........................ $36 \mathrm{~kg} .35$

Balles ......................... $3 \mathrm{~kg} .65$

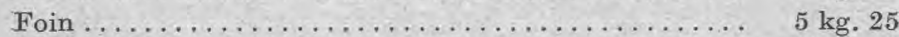

$2^{\circ}$ Composition du mélange concentré :

La même que celui de la période 9 .

Quantités de mélange concentré distribuées à chaque vache :

Premier groupe.

Numéro du sujet ...............

Quantité de mélange concentré (en kilogrammes) ..............

Valeur fourragère de la ration, re-

fus déduits (unités) ..........

Mat, album. digestibles (en grammes) $\ldots \ldots \ldots \ldots \ldots \ldots \ldots \ldots$

$\begin{array}{clllllll}32 & 33 & 29 & 31 & 30 & 34 & 2 \\ 7,4 & 5,0 & 5,2 & 9,0 & 3,7 & 2,6 & 2,6 \\ 12,7 & 10,7 & 10,8 & 13,7 & 9,6 & 8,7 & 8,7 \\ 1.504 & 1.147 & 1.164 & 1.709 & 942 & 786 & 762\end{array}$

Second groupe.

Numéro du sujet .................... 28

Quantité de mélange concentré (en kilogra mmes) . . . . . 10,0

Valeur fourragère de la ration, refus déduits (unités) ...

14,6

14,7 


\section{Période 11 (du ler au 8 Avril).}

$1^{\circ}$ Ration de base :

Betteraves ........................ $36 \mathrm{~kg} .35$

Balles ........................... $3 \mathrm{~kg} .65$

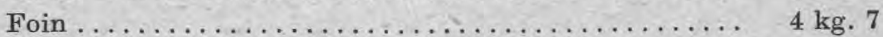

$2^{\circ}$ Composition du mélange concentré :

La même que celui de la période 9 .

Quantités de mélange concentré distribuées à chaque vache :

Premier groupe.

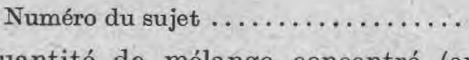

Quantité de mélange concentré (en kilogrammes) ...............

Valeur fourragère de la ration, refus déduits (unités) ............. Mat. album. digestibles (en grammes).

$\begin{array}{ccccccc}32 & 33 & 29 & 31 & 30 & 34 & 2 \\ 6,7 & 4,1 & 3,7 & 8,0 & 2,9 & 4,9 & 2,1 \\ 12,0 & 9,8 & 9,5 & 13,1 & 8,8 & 10,5 & 8,1 \\ 1.554 & 977 & 905 & 1.569 & 796 & 1.096 & 677\end{array}$

\section{Second groupe.}

Numéro du sujet $\ldots \ldots \ldots \ldots \ldots \ldots \ldots .28$

6

20

12

Quantité de mélange concentré (en kilogrammes) ...............

Valeur fourragère de la ration, refus déduits (unités) ..............

Mat.album, digestibles (en grammes).

$\begin{array}{cccccc}9,4 & 9,6 & 7,7 & 8,2 & 10,7 & 9,6 \\ 14,2 & 14,4 & 12,7 & 13,2 & 15,3 & 14,4 \\ 1.767 & 1.813 & 1.512 & 1.598 & 1.971 & 1.808\end{array}$

Période 12 (du 9 au 13 Avril).

10 Ration de base :

Betteraves ........................ $36 \mathrm{~kg} .35$

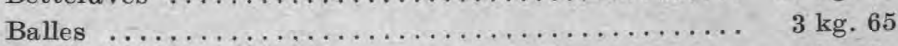

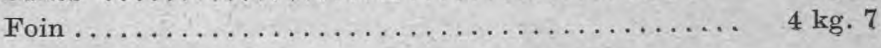

$2^{\circ}$ Composition du mélange concentré :

La même que celui de la période 9.

Quantités de mélange concentré distribuées à chaque vache :

Premier groupe.

Numéro du sujet

Quantité de mélange concentré (en

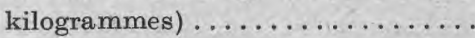

Valeur fourragère de la ration, refus déduits (unités) .............. Mat.album. digestibles (en grammes).
$33 \quad 29$

6,1

$9,5 \quad 9,2$

12,5

$\begin{array}{lll}923 & 879 & 1.466\end{array}$

30

34

2 $2,6 \quad 4,4 \quad 1,9$

$8,6 \quad 10,1 \quad 8,0$ $\begin{array}{lll}762 & 1.031 & 65\end{array}$ 
Second groupe.

Numéro du sujet 28

27

6

20

12

16

Quantité de mélange concentré (en kilogrammes) ...............

Valeur fourragère de la ration, refus déduits (unités) ..............

Mat.album. digestibles (en grammes).

$\begin{array}{llllll}8,5 & 8,8 & 7,0 & 7,4 & 9,7 & 8,8\end{array}$

$\begin{array}{llllll}13,5 & 13,7 & 12,2 & 12,6 & 14,5 & 13,7\end{array}$

$\begin{array}{llllll}1.648 & 1.672 & 1.413 & 1.484 & 1.826 & 1.684\end{array}$

Période 13 (du 14 au 22 Avril).

10 Ration de base :

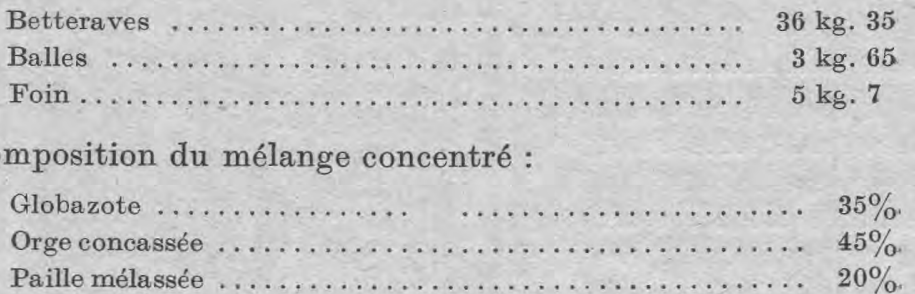

Quantités de mélange concentré distribuées à chaque vache :

Premier groupe.

Numéro du sujet ..................

kilogrammes) ................

Valeur fourragère de la ration, refus

déduits (unités) . . . . . . . . . . .

Mat.album. digestibles (en grammes).

$\begin{array}{ccccccc}32 & 33 & 29 & 31 & 30 & 34 & 2 \\ 5,4 & 2,9 & 2,4 & 5,9 & 2,1 & 3,8 & 2,0 \\ 11,7 & 9,4 & 9,0 & 12,2 & 8,8 & 10,7 & 8,6 \\ 1.372 & 914 & 835 & 1.360 & 789 & 1.072 & 757\end{array}$

\section{Second groupe.}

Numéro du sujet..$\ldots \ldots \ldots \ldots \ldots \ldots$

Quantité de mélange concentré (en kilogrammes) .................

Valeur fourragère de la ration, refus déduits (unités) ............... Mat. album. digestibles (en gra mmes).

$\begin{array}{cccccc}28 & 27 & 6 & 20 & 12 & 16 \\ 8,2 & 8,7 & 7,0 & 5,9 & 8,6 & 7,8 \\ 15,1 & 15,2 & 13,2 & 12,1 & 14,7 & 13,6 \\ 1.875 & 1.922 & 1.652 & 1.444 & 1.936 & 1.763 ;\end{array}$

Période 14 (du 23 au 26 Avril).

10 Ration de base :

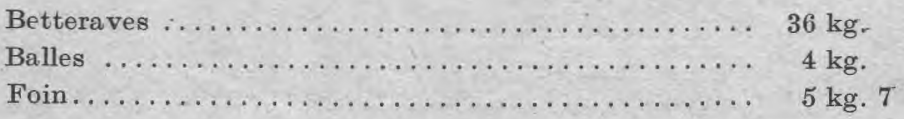

$2^{\circ}$ Composition du mélange concentré :

La même que celui de la période 13. 
Quantités de mélange concentré distribuées à chaque vache :

Premier groupe.

Numéro du sujet

$\begin{array}{rrrrrrr}32 & 33 & 29 & 31 & 30 & 34 & 2 \\ 6,6 & 3,8 & 3,4 & 5,1 & 3,1 & 5,1 & 2,3 \\ 12,8 & 10,3 & 9,9 & 13,4 & 9,3 & 11,5 & 9,6\end{array}$

Mat. album. digestibles (en grammes) $\ldots \ldots \ldots \ldots \ldots \ldots \ldots \ldots$

$\begin{array}{llllll}1.583 & 1.091 & 1.010 & 1.704 & 957 & 1.305\end{array}$

781

Second groupe.

Numéro du sujet $\ldots \ldots \ldots \ldots \ldots \ldots .28$

Quantité de mélange concentré (en kilogrammes) ..............

Valeur fourragère de la ration, refus déduits (unités) ............

Mat. album. digestibles (en grammes) $\ldots \ldots \ldots \ldots \ldots \ldots \ldots \ldots$
9,9

15,9

2.171

27

10,3

15,9

2.204

1.935

1.688

2.220

\section{PérIOde 15 (du 27 au 30 Avril).}

10 Ration de base :

Betteraves ........................ $35 \mathrm{~kg} .6$

Balles ........................ $4 \mathrm{~kg} .4$

Foin ......................... $5 \mathrm{~kg} .7$

20 Composition du mélange concentré :

La même que celui de la période 13.

Quantités de mélange concentré distribuées à chaque vache :

Premier groupe.

Numéro du sujet .............

Quantité de mélange concentré (en kilogrammes) ................

Valeur fourragère de la ration, refus déduits (unités) ............... Mat.album. digestibles (en grammes) .

$\begin{array}{ccccccc}32 & 33 & 29 & 31 & 30 & 34 & 2 \\ 5,4 & 2,9 & 2,4 & 5,9 & 2,1 & 3,9 & 2,0 \\ 11,9 & 9,6 & 9,2 & 12,9 & 8,9 & 7,3 & 8,4 \\ 1.389 & 936 & 860 & 1.479 & 803 & 1.113 & 745\end{array}$

Second groupe.

Numéro du sujet ..................

Quantité de mélange concentré (en kilogrammes) ................

Valeur fourragère de la ration, refus déduits (unités) ............... Mat.album. digestibles (en grammes) .

$\begin{array}{cccccc}28 & 27 & 6 & 20 & 12 & 16 \\ 8,2 & 8,7 & 7,0 & 5,9 & 8,6 & 7,8 \\ 14,4 & 14,3 & 13,3 & 11,8 & 14,8 & 13,5 \\ 1.898 & 1.941 & 1.675 & 1.432 & 1.960 & 1.780\end{array}$




\section{Période 16 (du $1^{\mathrm{er}}$ au 3 Mai).}

10 Ration de base :

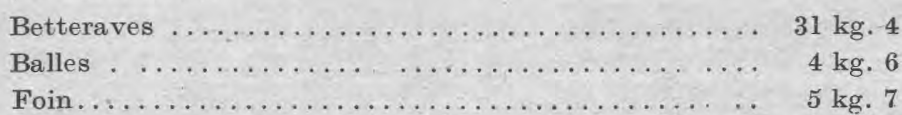

$2^{\circ}$ Composition du mélange concentré :

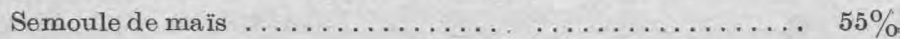

Orge concassée........................ $25 \%$

Paille mélassée ........................ $20 \%$

Quantités de mélange concentré distribuées à chaque vache :

Premier groupe.

Numéro du sujet $\ldots \ldots \ldots \ldots \ldots \ldots \ldots$.

grammes) $\ldots \ldots \ldots \ldots \ldots \ldots \ldots \ldots \ldots \quad 5,2 \quad 3,0 \quad 2,3 \quad 5,8 \quad 2,0 \quad 4,4 \quad 1,2$

Valeur fourragère de la ration, refus dé-

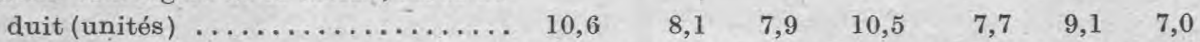

$\begin{array}{lllllllll}\text { Mat.album. digestibles (en grammes) } & \ldots & 1.042 & 686 & 657 & 1.066 & 628 & 845 & 539\end{array}$

Second groupe.

Numéro du sujet ................. $28 \quad 27$

Quantité de mélange concentré (en kilo-

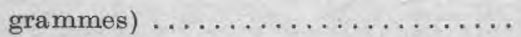

Valeur fourragère de la ration, refus dé-

duits (unités) $\ldots \ldots \ldots \ldots \ldots \ldots \ldots \ldots 13,1 \quad 10,3 \quad 11,6 \quad 8,9 \quad 11,9 \quad 10,4$

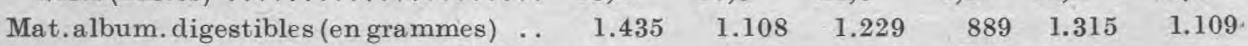

Période 17 (du 4 au 6 Mai).

$1^{\circ}$ Ration de base :

Betteraves ......................... $33 \mathrm{~kg} .1$

Balles ...................... $3 \mathrm{~kg} .9$

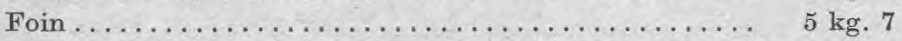

$2^{\circ}$ Composition du mélange concentré :

La même que celui de la période 16 .

Quantités de mélange concentré distribuées à chaque vache :

Premier groupe.

Numéro du sujet $\ldots \ldots \ldots \ldots \ldots \ldots$

Quantité de mélange concentré (en kilogrammes) ...................

Valeur fourragère de la ration, refus déduits (unités) . . . . . . . . . . . .

Mat.album. digestibles (en grammes) .. $32 \quad 33$

$\begin{array}{lllllll}6,5 & 4,1 & 3,2 & 7,1 & 3,0 & 5,5 & 2,7\end{array}$

$\begin{array}{lllllll}11,0 & 8,0 & 7,7 & 11,4 & 7,9 & 9,7 & 7,6\end{array}$

$\begin{array}{lllllll}1.133 & 694 & 660 & 1.207 & 688 & 992 & 662\end{array}$


Second groupe.

Numéro du sujet $\ldots \ldots \ldots \ldots \ldots \ldots \ldots, \quad 28 \quad 27$

$6 \quad 20 \quad 12$

16

Quantité de mélange concentré (en kilo-

grammes) $\ldots \ldots \ldots \ldots \ldots \ldots \ldots \ldots \ldots \quad 10,5 \quad 10,5 \quad 9,7 \quad 8,5 \quad 12,2 \quad 10,5$

Valeur fourragère de la ration, refus dé-

duits (unités) $\ldots \ldots \ldots \ldots \ldots \ldots \ldots \ldots 12,5 \quad 11,2 \quad 11,2 \quad 9,6 \quad 12,2 \quad 10,9$

$\begin{array}{llllllll}\text { Mat.album. digestibles (en grammes) } & \ldots & 1.367 & 1.216 & 1.160 & 981 & 1.359 & 1.157\end{array}$

RÉSULTATS DU GONTROLE DE RENDEMENT

(Variation du rendement en lait ramené à $4 \%$ de matière grasse, et variation du poids vif.)

PREMIER GROUPE

Vache No 32.

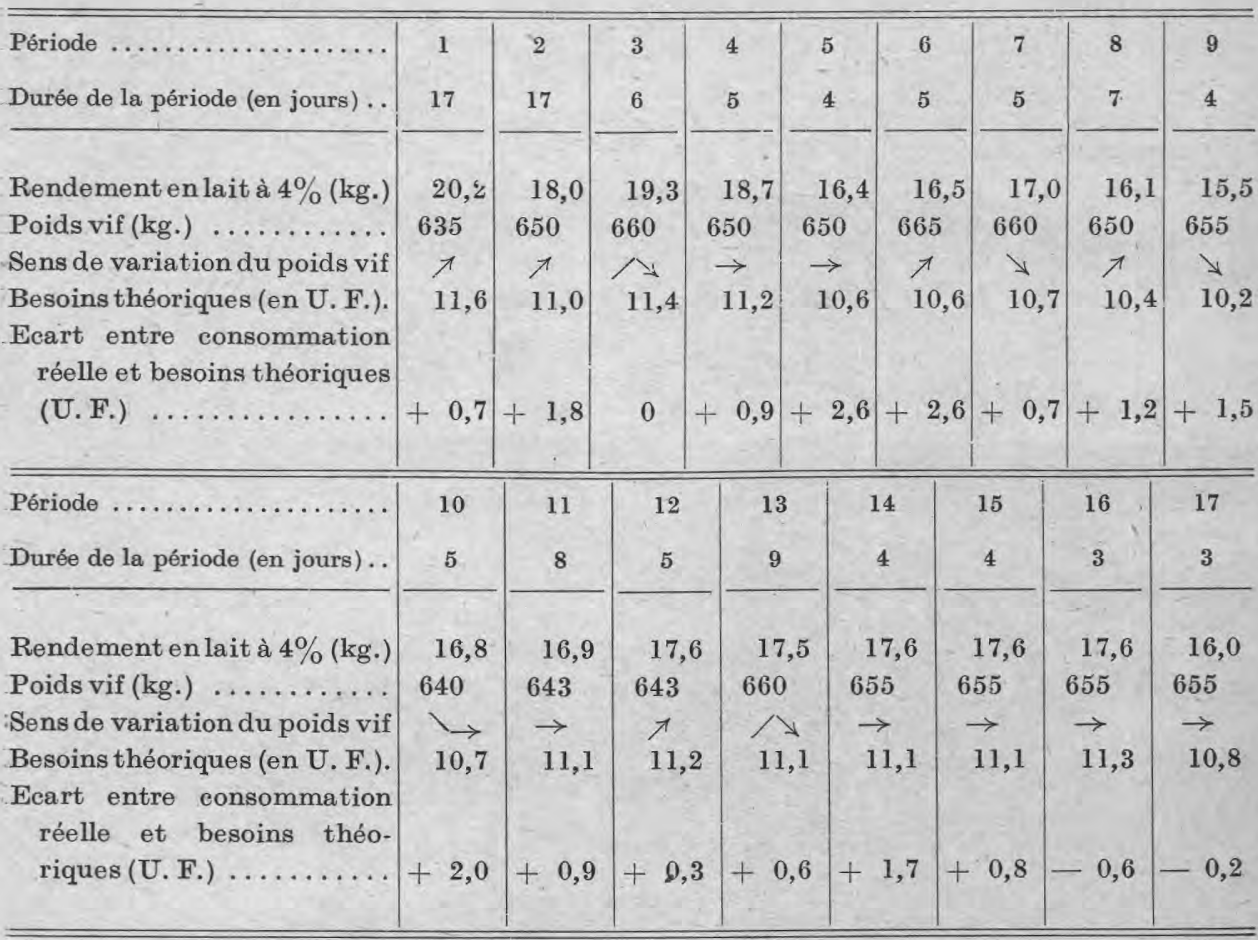


Vache No 33.

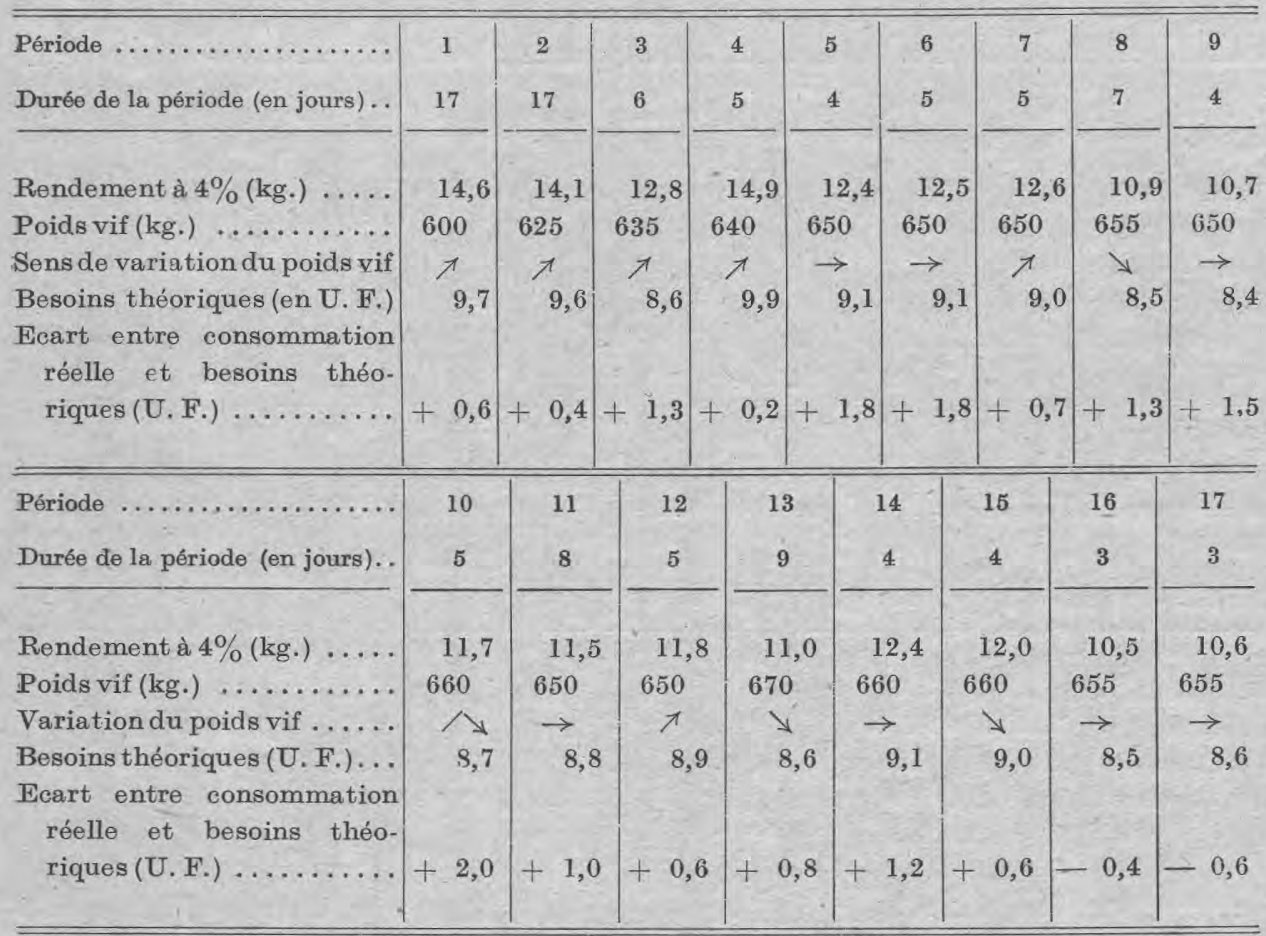

\section{Vache No 29.}

\begin{tabular}{|c|c|c|c|c|c|c|c|c|c|}
\hline Période $\ldots \ldots \ldots \ldots \ldots \ldots \ldots$ & 1 & 2 & 3 & 4 & 5 & 6 & 7 & 8 & 9 \\
\hline Durée de la période (en jours)... & 17 & 17 & 6 & 5 & 4 & 5. & 5 & 7 & 4 \\
\hline Rendement à $4 \%$ (kg.) .... & 14,9 & 12,5 & 13,1 & 12,1 & 12,2 & 12,1 & 12,7 & 10,8 & 11,7 \\
\hline Poids vif (kg.) . . . . . . . . & 630 & 615 & 630 & 630 & 635 & 640 & 640 & 650 & 645 \\
\hline Variation du poids vif ...... & $\searrow$ & $\pi$ & $\rightarrow$ & $\nearrow$ & $\pi$ & $\rightarrow$ & $\nearrow$ & $\searrow$ & $\searrow$ \\
\hline Besoins théoriques (U. F.)... & 9,7 & 8,9 & 9,1 & 8,8 & 8,8 & 8,8 & 9,0 & 8,3 & 8,6 \\
\hline $\begin{array}{l}\text { Ecart entre eonsommation } \\
\text { réelle et besoins théo- } \\
\text { riques (U. F.) } \ldots \ldots \ldots \ldots\end{array}$ & $\begin{array}{r} \\
+\quad 0,6\end{array}$ & $+0,9$ & $+0,2$ & $+1,0$ & $+1,8$ & $+1,7$ & $+0,4$ & $+1,6$ & $+1,4$ \\
\hline
\end{tabular}


A. M. LEROY. - CONTRIBUTION A L'ÉTUDE

\begin{tabular}{|c|c|c|c|c|c|c|c|c|}
\hline Période...$\ldots \ldots \ldots \ldots \ldots$ & 10 & 11 & 12 & 13 & 14 & 15 & 16 & 17 \\
\hline Durée de la période (en jours).. & 5 & 8 & 5 & 9 & 4 & 4 & 3 & 3 \\
\hline Rendement à $4 \%$ (kg.) . . . . . & 11,2 & 11,2 & 10,1 & 11,4 & 11,8 & 10,2 & 7,8 & 9,3 \\
\hline Poids vif (kg.) $\ldots \ldots \ldots \ldots$ & 635 & 635 & 635 & 640 & 635 & 635 & 635 & 630 \\
\hline Variation du poids vif ...... & $\rightarrow$ & $\rightarrow$ & $\rightarrow$ & 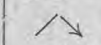 & $\rightarrow$ & $\rightarrow$ & $\searrow$ & $\searrow$ \\
\hline Besoins théoriques (U. F.) ... & 8,5 & 8,5 & 8,1 & 8,6 & 8,7 & 8,1 & 7,3 & 7,8 \\
\hline $\begin{array}{l}\text { Ecart entre consommation } \\
\text { réelle et besoins théo- } \\
\text { riques (U.F.) . . . . . . }\end{array}$ & $\begin{array}{r} \\
+\quad 2,3\end{array}$ & $+1,0$ & $+1,1$ & $+0,4$ & $+1,2$ & $+1,1$ & $+0,6$ & $-0,1$ \\
\hline
\end{tabular}

\section{Vache $N^{\circ} 31$.}

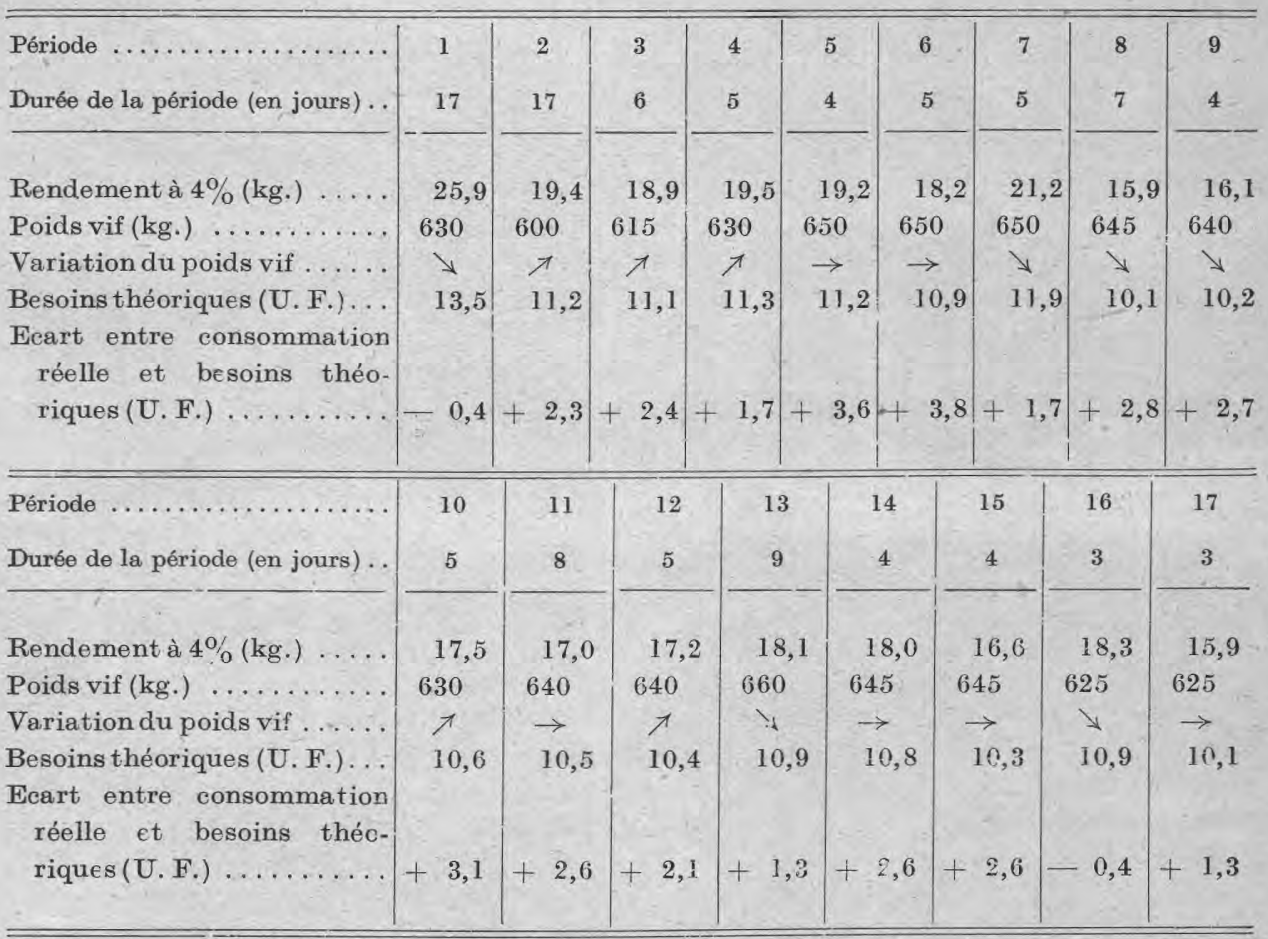


Vache $N^{\circ} 30$

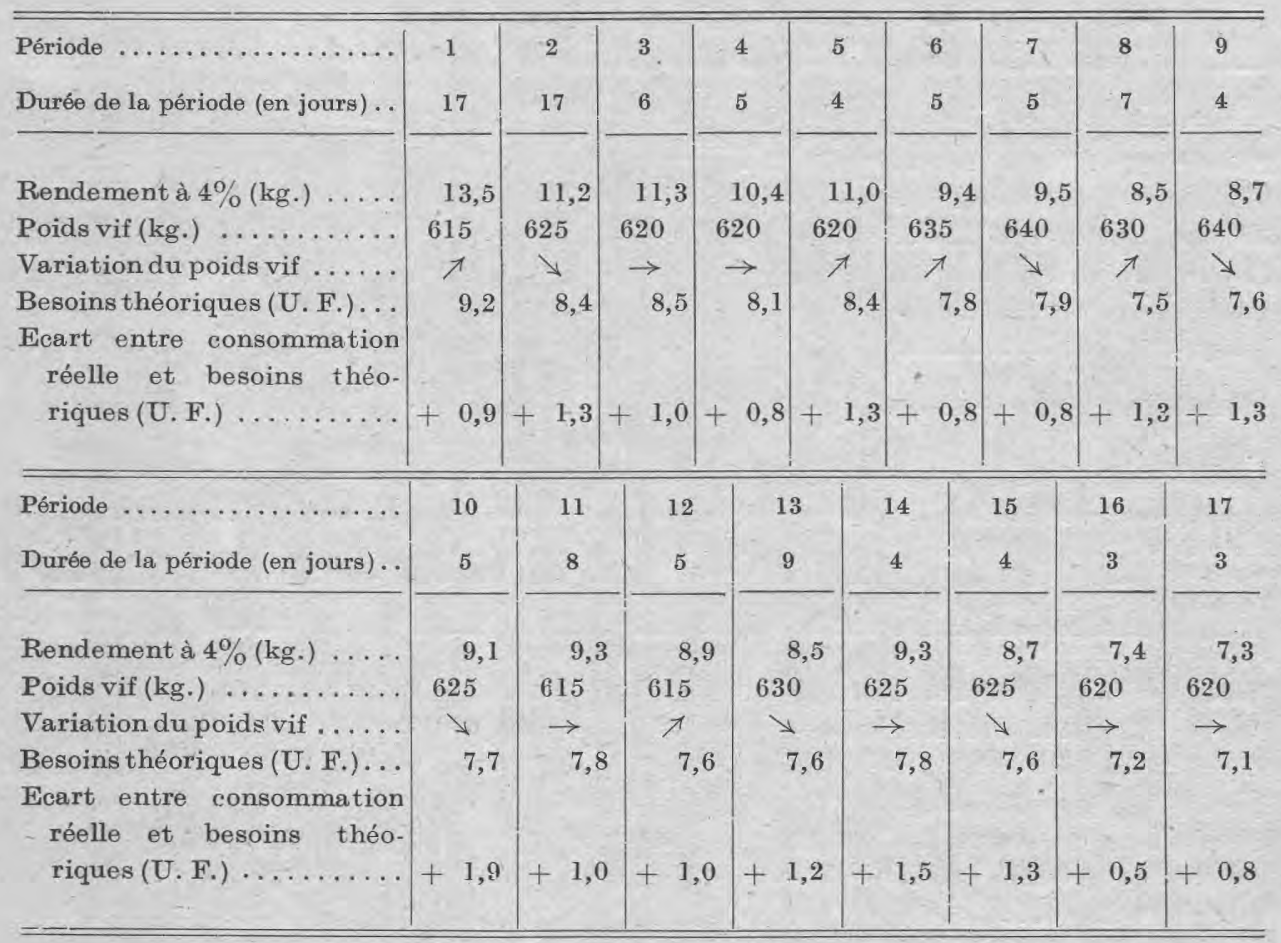

\section{Vache No 34.}

\begin{tabular}{|c|c|c|c|c|c|c|c|c|c|}
\hline Période $. . . \ldots \ldots \ldots \ldots \ldots . . . \ldots$ & 1 & 2 & 3 & 4 & 5 & 6 & 7 & 8 & 9 \\
\hline Durée de la période (en jours).. & 17 & 17 & 6 & 5 & 4 & 5 & 5 & 7 & 4 \\
\hline Rendement à $4 \%$ (kg.) ..... & 18,7 & 16,1 & 16,3 & 13,4 & 16,0 & 16,4 & 17,4 & 16,3 & 16,3 \\
\hline Poids vif (kg.) ........... & 570 & 580 & 580 & 590 & 600 & 605 & 600 & 605 & 610 \\
\hline Variation du poids vif ...... & $\pi$ & $\rightarrow$ & $\pi$ & $\pi$ & $\nearrow$ & $\searrow$ & $\rightarrow$ & $\pi$ & $\searrow$ \\
\hline Besoins théoriques (U. F.)... . & 10,9 & 10,0 & 10,1 & 9,1 & 10,1 & 10,2 & 10,6 & 10,2 & 10,2 \\
\hline $\begin{array}{l}\text { Ecart entre consommation } \\
\text { réelle et besoins théo- } \\
\text { riques (U.F.) } \ldots \ldots \ldots \ldots \ldots\end{array}$ & $\begin{array}{r}1 \\
+\quad 0,3 \\
\end{array}$ & $+1,5$ & $+0,7$ & $+1,2$ & $+1,4$ & $+1,2$ & $-0,6$ & $+0,1$ & $+0,1$ \\
\hline
\end{tabular}


A. M. LEROY. - CONTRIBUTION A L'ÉTUDE

\begin{tabular}{|c|c|c|c|c|c|c|c|c|}
\hline Période $. . . \ldots \ldots \ldots \ldots \ldots . . . . .$. & 10 & 11 & 12 & 13 & 14 & 15 & 16 & 17 \\
\hline Durée de la période (en jours) & 5 & 8 & 5 & 9 & 4 & 4 & 3 & 3 \\
\hline Rendement à $4 \%$ (kg.) . . . . . & 16,0 & 14,9 & 16,9 & 14,8 & 18,5 & 16,4 & 15,0 & 14,9 \\
\hline Poids vif (kg.) $\ldots \ldots \ldots \ldots$ & 600 & 600 & 600 & 610 & 600 & 600 & 595 & 595 \\
\hline Variation $d u$ poids vif $\ldots .$. & $\rightarrow$ & $\rightarrow$ & $\rightarrow$ & $\triangle$ & $\rightarrow$ & $\searrow$ & $\searrow$ & $\rightarrow$ \\
\hline Besoins théoriques (U. F.)... & 10,1 & 9,8 & 10,4 & 9,5 & 11,0 & 10,3 & 10,0 & 10,0 \\
\hline $\begin{array}{l}\text { Ecart entre consommation } \\
\text { réelle et besoins théo- } \\
\text { riques (U. F.) } \ldots \ldots \ldots \ldots\end{array}$ & $-1,3$ & $+0,7$ & $-0,3$ & $+1,2$ & $+0,0$ & $-3,0$ & $-0,9$ & $-0,3$ \\
\hline
\end{tabular}

Vache No 2.

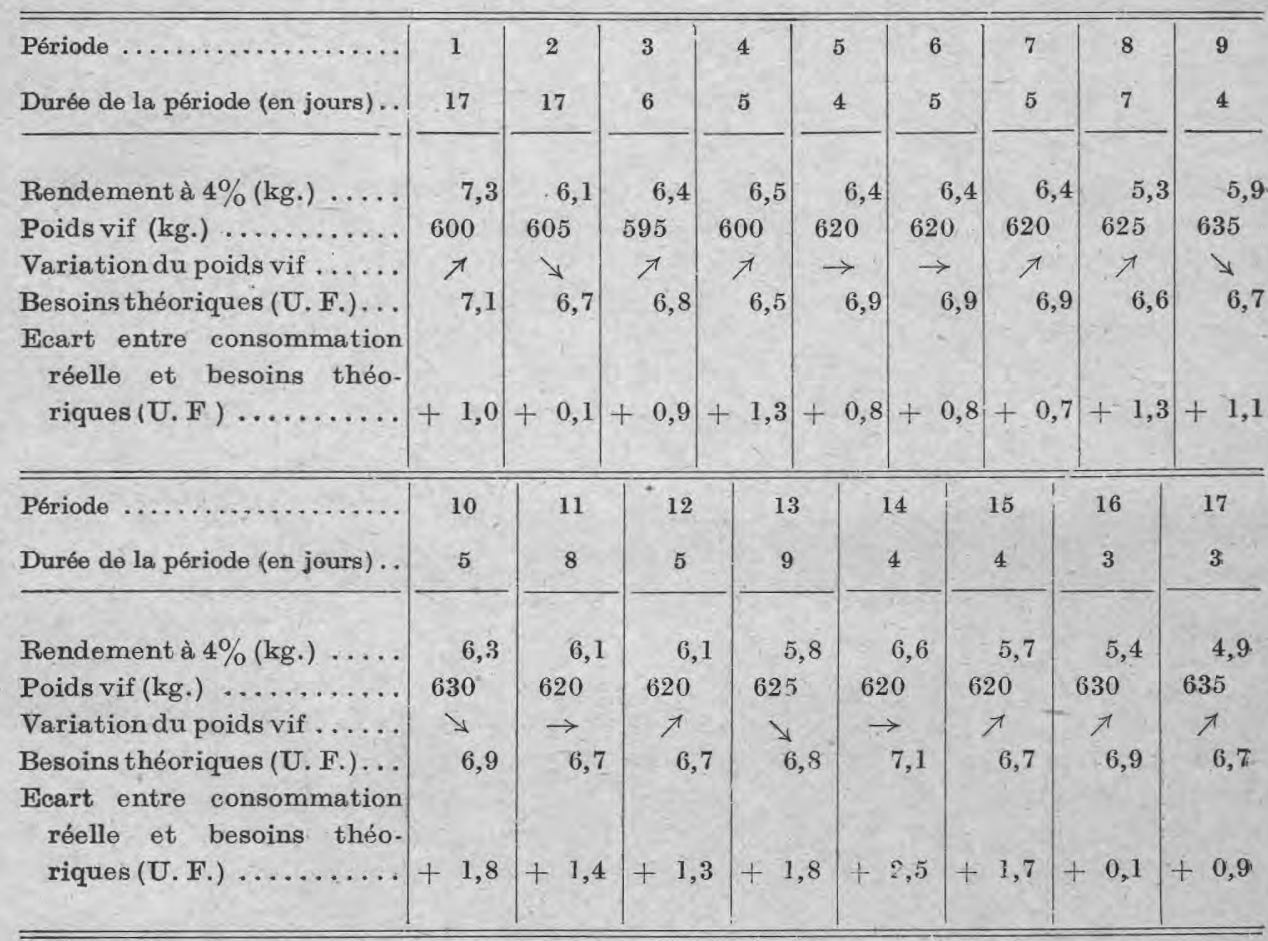




\section{SECOND GROUPE}

Vache $N^{\circ} 28$.

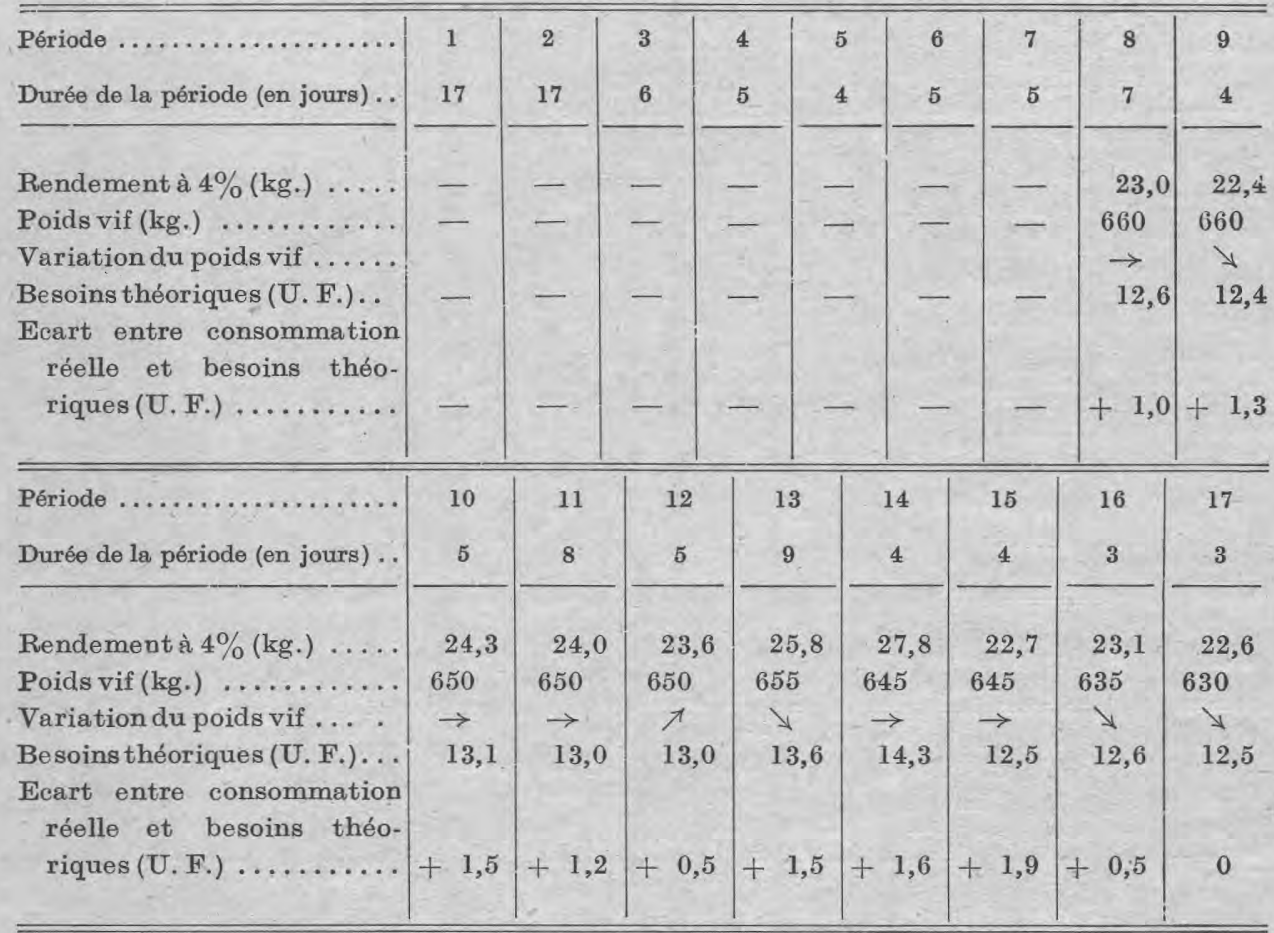

Vache $N^{\circ} 27$.

\begin{tabular}{|c|c|c|c|c|c|c|c|c|c|c|}
\hline Période..,$\ldots \ldots$. & 8 & 9 & 10 & 11 & 12 & 13 & 14 & 15 & 16 & 17. \\
\hline $\begin{array}{c}\text { Durée de la période (en } \\
\text { jours) } \ldots \ldots \ldots \ldots \ldots \ldots\end{array}$ & 7 & 4 & 5 & 8 & 5 & 9 & 4 & 4 & 3 & 3 \\
\hline \multicolumn{11}{|l|}{ Rendement en lait } \\
\hline à $4 \%$ (kg.) ...... & 22,2 & 20,7 & 21,7 & 24,6 & 25,0 & 24,5 & 25,1 & 22,7 & 22,8 & 20,5 \\
\hline Poids vif (kg.) .... . & 630 & 640 & 630 & 630 & 630 & 645 & 625 & 625 & 610 & 600 \\
\hline $\begin{array}{l}\text { Variation du poids } \\
\text { vif ............. }\end{array}$ & $\pi$ & $\searrow$ & $\rightarrow$ & $\rightarrow$ & $\pi$ & $\searrow$ & $\rightarrow$ & $\searrow$ & $\searrow$ & $\searrow$ \\
\hline $\begin{array}{c}\text { Besoins théoriques } \\
\text { (U.F.) } \ldots \ldots \ldots \ldots\end{array}$ & 12,2 & 11,7 & 12,1 & 13,1 & 13,2 & 13,0 & 13,2 & 12,4 & 12,4 & 11,7 \\
\hline $\begin{array}{l}\text { Ecart entre consom- } \\
\text { mation réalle et } \\
\text { besoins théoriques }\end{array}$ & & & & & & & & & & \\
\hline$(U, F.) \ldots \ldots \ldots$ & $+1,6$ & $+2,1$ & $+2,6$ & $+1,3$ & $-1,1$ & $+2,2$ & $+2,7$ & $+1,9$ & $-2,1$ & $-0,5$ \\
\hline
\end{tabular}


Vache No 6.

\begin{tabular}{|c|c|c|c|c|c|c|c|c|c|c|}
\hline $\begin{array}{l}\text { Période } \ldots \ldots \ldots \ldots \ldots \\
\text { Durée de la période (en } \\
\text { jours) } \ldots \ldots \ldots \ldots \ldots\end{array}$ & 8 & 9 & 10 & 11 & 12 & 13 & 14 & 15 & 16 & 17 \\
\hline $\begin{array}{l}\text { Rendement en lait } \\
\text { à } 4 \% \text { (kg.) } \ldots \ldots\end{array}$ & 18,2 & 17,3 & 19,6 & 23,1 & 19,7 & 21 & 21,8 & 20,9 & 18,9 & 18,1 \\
\hline $\begin{array}{l}\text { Poids vif (kg.) } \ldots . . \\
\text { Variation du poids }\end{array}$ & 665 & 670 & 665 & 670 & 670 & 695 & 680 & 680 & 675 & 675 \\
\hline $\begin{array}{r}\text { vif ............ } \\
\text { Besoins théoriques }\end{array}$ & $\pi$ & $\searrow$ & $\pi$ & $\rightarrow$ & $\pi$ & $\searrow$ & $\pi$ & $\searrow$ & $\searrow$ & $\rightarrow$ \\
\hline $\begin{array}{l}\text { (U. F.) . . . . . . } \\
\text { Ecart entre consom- } \\
\text { mation réelle et } \\
\text { besoins théoriques } \\
\text { (U. F.) . . . . . . }\end{array}$ & $+\quad 1,2$ & $+1,5$ & $+1,7$ & $-12,7$ & $+1,0$ & $+1,2$ & 12,3 & $+1,3$ & $+0,3$ & $+0,1$ \\
\hline
\end{tabular}

Vache $N^{\circ} 20$.

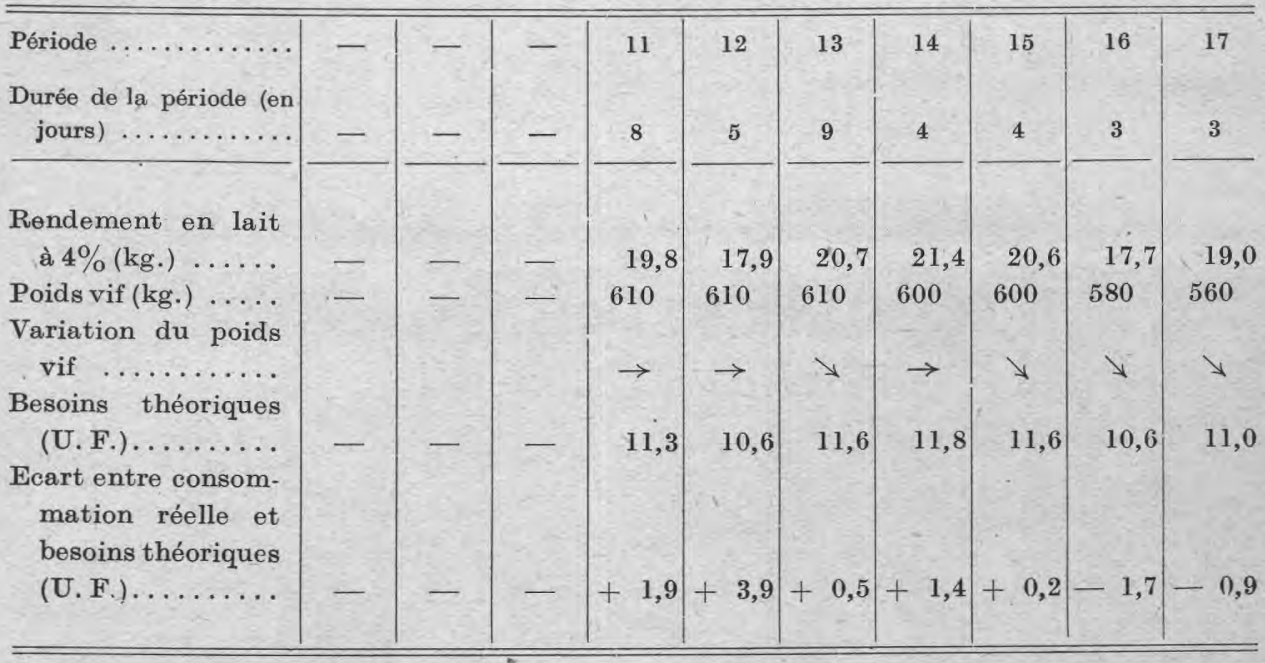


Vache $N^{\circ} 12$.

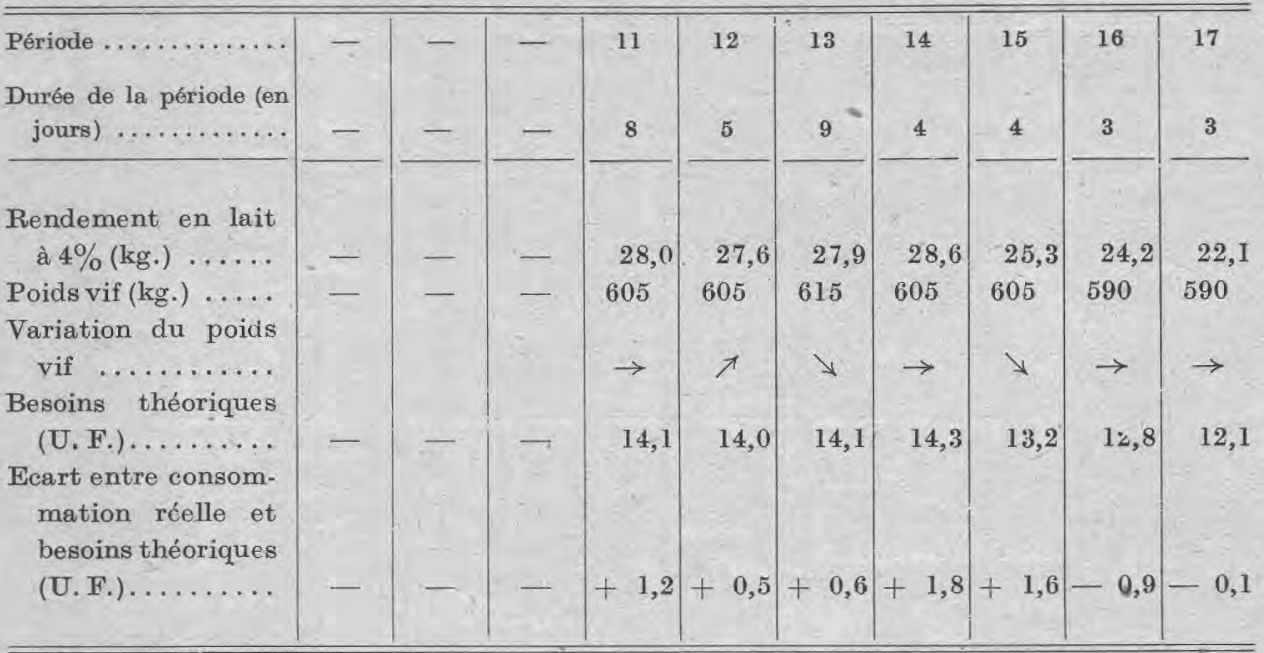

Vache No 16.

\begin{tabular}{|c|c|c|c|c|c|c|c|c|c|c|}
\hline Période ............. & - & - & - & 11 & 12 & 13 & 14 & 15 & 16 & 17 \\
\hline $\begin{array}{c}\text { Durée de la période (en } \\
\text { jours).............. }\end{array}$ & - & - & - & 8 & 5 & 9 & 4 & 4 & 3 & 3 \\
\hline Rendement en lait & & & & & & & & & & \\
\hline (kg.) ..... & - & - & 一 & 24,1 & 24,8 & 25,3 & 25,8 & 24,9 & 22,6 & 21,3 \\
\hline $\begin{array}{l}\text { Poids vif (kg.) } \ldots . . \\
\text { Variation du poids }\end{array}$ & - & - & - & 605 & 605 & 615 & 600 & 600 & 580 & 580 \\
\hline $\begin{array}{r}\text { vif ............. } \\
\text { Besoins théoriques }\end{array}$ & & & & $\rightarrow$ & $\pi$ & $\searrow$ & $\rightarrow$ & $y$ & $\rightarrow$ & $\rightarrow$ \\
\hline $\begin{array}{l}\text { (U. F.) .......... } \\
\text { Ecart entre consom. } \\
\text { mation réelle et } \\
\text { besoins théoriques }\end{array}$ & - & - & - & 12,7 & 12,9 & 13,1 & 13,3 & 13,0 & 12,2 & 11,8 \\
\hline (U. F.)......... & - & - & - & $+1,7$ & $+0,8$ & $+0,5$ & $+1,7$ & $-0,5$ & $-1,8$ & $-0,9$ \\
\hline
\end{tabular}

A l'aide de la documentaion qui précède, il a été possible de calculer les résultats moyens fournis par chaque groupe d'animaux, et pour l'ensemble des périodes. Ces résultats figurent sur le tableau ci-dessous.

Pour la détermination des gains ou pertes de poids vif, nous nous sommes servis de la méthode des moindres carrés, qui permet de calculer l'équation de la droite autour de laquelle se produisent normalement les variations cycliques du poids, ainsi que l'a montré, dans son intéressant travail, le Professeur Maymone. 


\section{RÉSULTATS GÉNÉRAUX DES EXPÉRIENGES}

\begin{tabular}{|c|c|c|c|c|c|}
\hline Désignation de l'expérience & $\begin{array}{c}\text { Valeur } \\
\text { fourragère } \\
\text { de la } \\
\text { ration } \\
\text { réellement } \\
\text { consom- } \\
\text { mée } \\
\text { Unités }\end{array}$ & $\begin{array}{l}\text { Lait } \\
\text { recueilli } \\
\text { ramené à } \\
4 \% \text { de } \\
\text { matière } \\
\text { grasse } \\
\\
\text { Kilo- } \\
\text { grammes }\end{array}$ & $\begin{array}{c}\text { Kilo- } \\
\text { grammes }\end{array}$ & $\begin{array}{c}\text { Variation } \\
\text { quotidienne } \\
\text { du poids } \\
\text { vif } \\
\text { Kilogrammes }\end{array}$ & $\begin{array}{l}\text { Solde } \\
\text { disponi- } \\
\text { ble pour } \\
\text { la pro- } \\
\text { duction } \\
\text { (ration } \\
\text { d'en- } \\
\text { tretien } \\
\text { déduite) } \\
\text { Unités }\end{array}$ \\
\hline Gournay-sur-Marne ..... & 10,3 & & 619 & & 5,7 \\
\hline Quiévy $\ldots \ldots \ldots \ldots \ldots$ & 14,6 & 27,2 & 682 & $-0,190$ & 9,7 \\
\hline Bonnelles (vaches moy.) .. & 10,45 & 13,3 & 632 & $+0,246$ & 5,8 \\
\hline $\begin{array}{l}\text { Bonnelles (vaches à grand } \\
\text { rendement) } \ldots \ldots \ldots \ldots\end{array}$ & 13,9 & 23,8 & 632 & $+0,102$ & 9,25 \\
\hline
\end{tabular}

Les données de la dernière colonne de droite de ce tableau ont été obtenues en retranchant des valeurs fourragères des rations réellement consommées les quantités correspondant aux rations d'entretien.

Désignons maintenant par $x$ la ration de production par kilogramme de lait produit, et par $y$, la ration de croissance correspondant à une variation de poids vif d'un kilogramme. Nous pouvons obtenir le système d'équations à deux inconnues ci-après :

I Pour Gournay-sur-Marne $\ldots \quad 12,25 x+0,297 y=5,7$ unités.

II Pour Quiévy $\ldots \ldots \ldots \ldots \ldots$ 27,2 $x-0,190 y=9,7$ unités.

III Pour Bonnelles (vaches moyen-

$$
\text { nes) } \ldots \ldots \ldots \ldots \ldots \ldots \ldots \ldots \ldots \ldots \ldots \ldots \text { 13,3 } x+0,246 y=5,8 \text { unités. }
$$

IV Pour Bonnelles (vaches à

$$
\text { grand rendement) } \ldots \ldots \ldots 23,8 \quad x+0,102 y=9,25 \text { unités. }
$$

Il ne saurait y avoir, bien entendu, une seule couple de données $x$ et $y$ formant la solution de ce système complexe. Mais on peut combiner les équations deux à deux, par exemple I avec III, III avec IV, II avec IV, etc. On obtiendrait dans ce cas les solutions ci-après :

En résolvant I et III $\ldots \ldots . \quad x=0,372$

$$
\begin{aligned}
& y=3,85 \\
& y=3,12 \\
& y=3,48
\end{aligned}
$$$$
\text { En résolvant III et IV .... } x=0,381
$$ 


\begin{tabular}{|c|c|c|}
\hline En résolvant I et II & $\ldots \ldots \ldots=x=0,373$ & $y=3,37$ \\
\hline En résolvant II et IV & $\ldots \ldots \quad x=0,378$ & $y=2,80$ \\
\hline En résolvant I et IV & $x=0,372$ & $y=3,89$ \\
\hline Valeurs moyennes... & $x=0,375$ & $y=3,42$ \\
\hline
\end{tabular}

On voit qu'il existe une remarquable concordance entre les valeurs de $x$ fournies par ces divers systèmes. Les valeurs de $y$, beaucoup plus variables, oscillent cependant autour de la valeur moyenne 3,42. En particulier, nous croyons devoir insister sur le fait que le système des équations I et III, qui se rapporte aux vaches de moyenne production, donne une valeur de $x$ presque identique à celle du système II et IV, correspondant aux vaches à grand rendement. Les tables d'alimentation conservent donc la même valeur pratique, aussi bien lorsqu'il s'agit de calculer des rations pour vaches à 30 kilogrammes de lait par jour que dans le cas de laitières moyennes. La ration de production est done bien très voisine de 0,4 unité fourragère par kilogramme de lait à $4 \%$, ce qui confirme les données récemment publiées par le regretté Professeur danois Frederiksen.

Pour cette raison, nous avons modifié en conséquence le tableau donnant la ration de produetion laitière des vaches contenu dans la notice sur les Equivalents Fourragers de la Société Nationale d'Encouragement à l'Agrieulture ; les éditions de cette publication, à partir de 1933, diffèrent ainsi légèrement sur ce point des précédentes, et se rapprochent davantage de la réalité.

$\mathrm{Si}$ nous admettons maintenant que la ration de croissance est la même pour toutes les vaches, et possède la valeur de 3 u. 5 (1) par kilogramme de gain ou de perte de poids, nous pouvons ealeuler pour chacune des vaches la ration de production laitière correspondant à un kilogramme de lait, et mesurer ainsi l'importance des variations individuelles. En réalité, nous nous livrons à un calcul approximatif, car il est presque certain que la ration de croissance par unité de poids est un caractère individuel, au même titre que la ration de production laitière, dont il serait logique de se préoccuper. Mais comme les écarts de poids vif quotidien sont relativement faibles, nous pouvons admettre que la correction qu'il y aurait lieu d'apporter dans chaque cas afin de tenir compte de la variabilité de l'indice correspondant à la ration de croissance est d'importance négligeable. Il est d'ailleurs facile de montrer qu'il en est bien ainsi :

Considérons le cas de la vache Simplon, qui, pendant toute la

(1) Ce nombre est obtenu en arrondissant au dixième supérieur le résultat trouvé par le calcul qui précède, 3 u. 42. 
durée de l'expérience de Gournay, s'est trouvée dans les conditions ci-après :

\begin{tabular}{|c|c|c|c|c|c|}
\hline $\begin{array}{l}\text { Poids } \\
\text { moyen }\end{array}$ & $\begin{array}{c}\text { Unités } \\
\text { fourragères } \\
\text { consom- } \\
\text { mées }\end{array}$ & $\begin{array}{c}\text { Variations } \\
\text { quotidiennes } \\
\text { du } \\
\text { poids vif }\end{array}$ & $\begin{array}{l}\text { Produc- } \\
\text { tion } \\
\text { laitière } \\
\text { en } \\
\text { kilo- } \\
\text { grammes } \\
\text { à } 4 \%\end{array}$ & $\begin{array}{c}\text { Ration } \\
\text { d'entre- } \\
\text { tien }\end{array}$ & $\begin{array}{l}\text { dispo- } \\
\text { nible } \\
\text { pour la } \\
\text { produe- } \\
\text { tion } \\
\text { laitière } \\
\text { et le } \\
\text { gain de } \\
\text { poids }\end{array}$ \\
\hline
\end{tabular}

$581 \mathrm{~kg}$. $\quad 10 \mathrm{u} .34+0 \mathrm{~kg} .223 \quad 13 \mathrm{~kg} .1 \quad 4 \mathrm{u} .4 \quad 5 \mathrm{u} .94$

Dans l'hypothèse d'un indice de croissance constant et égal à 3 u. 5 par kilogramme, la correction pour variation de poids vif est de $3,5 \times 0,223=0 \mathrm{u}$. 78 , et la valeur de la ration de production par kilogramme de lait s'obtient par le calcul suivant :

$$
\frac{5,94-0,78}{13,1}=0 \text { u. } 394 \text {. }
$$

Supposons maintenant que l'indice individuel de croissance correspondant à la vache Simplon soit de 4 unités; on aurait alors une correction de 0 u. 89 , et la nouvelle valeur de la ration de production laitière serait de $\frac{5,94-0,89}{13,1}=0 \mathrm{u}$. 386, c'est-à-dire très voisine de la précédente.

Les résultats des calculs des indices individuels de production laitière, corrigés de l'influence de la variation du poids vif, avec indice de correction de 3 u. 5 par kilogramme de gain ou de perte de poids, figurent dans le tableau ci-dessous. Les données de ce tableau qui correspondent aux variations individuelles de poids vif ont toutes été obtenues par la méthode des moindres carrés, avec l'aide des poids individuels observés aux diverses périodes des expériences.

\section{DÉTERMINATION DES RATIONS INDIVIDUELLES DE PRODUGTION LAITIËRE \\ (par kilogramme de lait).}

A. EXPÉRIENCES DE GOURNAY-SUR-MARNE

Valeurs fourragères

\begin{tabular}{|c|c|c|c|c|c|c|c|c|}
\hline $\begin{array}{l}\text { Désignation } \\
\text { de l'animal }\end{array}$ & $\begin{array}{l}\text { Poids } \\
\text { moyen }\end{array}$ & $\begin{array}{l}\text { Lait } \\
\text { aे } 4 \%\end{array}$ & $\begin{array}{l}\text { U. F. } \\
\text { con- } \\
\text { som- } \\
\text { mées }\end{array}$ & $\begin{array}{l}\text { Variations } \\
\text { quoti- } \\
\text { diennes de } \\
\text { poids vif }\end{array}$ & $\begin{array}{l}\text { de } \\
\text { l'en- } \\
\text { tre- } \\
\text { tien }\end{array}$ & $\begin{array}{l}\text { corres- } \\
\text { pondant } \\
\text { aux } \\
\text { varia- } \\
\text { tions du } \\
\text { poids vif }\end{array}$ & $\begin{array}{l}\text { dispo- } \\
\text { nibles } \\
\text { pour } \\
\text { le lait }\end{array}$ & $\begin{array}{l}\text { U. F. } \\
\text { par } \\
\text { kilo- } \\
\text { gram- } \\
\text { me de } \\
\text { lait }\end{array}$ \\
\hline & $\mathrm{Kg}$. & $\mathrm{Kg}$. & Unités & $\mathrm{Kg}$. & Unités & Unités & Unités & \\
\hline plon .. & 581 & 13,1 & 10,34 & $+0,223$ & 4,4 & $+0,78$ & 5,16 & 0,394 \\
\hline & 621 & 13,2 & 10,27 & $+0,421$ & 4,6 & $+1,47$ & 4,20 & 0,318 \\
\hline & 679 & 12,6 & 10,82 & $+0,354$ & 4,9 & $+1,24$ & 4,68 & 0,372 \\
\hline taigne & 594 & 9,9 & 9,84 & $+0,058$ & 4,5 & $+0,20$ & 5,16 & 0,520 \\
\hline
\end{tabular}




\section{B. EXPÉRIENCES DE QUIÉVY}

Leeuwarder

$\begin{array}{rrrrrrrrr}\text { Gérard . . . } & 620 & 23,8 & 13,40 & +0,313 & 4,6 & +1,48 & 7,32 & 0,308 \\ \text { Bonne Mère } \ldots & 808 & 25,1 & 14,44 & -0,905 & 5,45 & -3,17 & 12,16 & 0,485 \\ \text { Same IV . . . . } & 607 & 30,8 & 15,75 & -0,004 & 4,55 & -0,01 & 11,20 & 0,364 \\ \text { Detje........ } & 694 & 30,4 & 15,65 & -0,220 & 4,97 & -0,77 & 11,45 & 0,376\end{array}$

C. EXPERIENCES DE BONNELLES - VACHES MOYENNES

$\begin{array}{rrrrrrrrr}32 \ldots \ldots \ldots \ldots & 651 & 17,4 & 12,17 & +0,099 & 4,75 & +0,35 & 7,07 & 0,406 \\ 33 \ldots \ldots \ldots \ldots & 645 & 12,4 & 10,10 & +0,559 & 4,72 & +1,96 & 3,42 & 0,276 \\ 29 \ldots \ldots \ldots \ldots & 636 & 12,0 & 9,88 & +0,150 & 4,68 & +0,52 & 4,68 & 0,390 \\ 31 \ldots \ldots \ldots \ldots & 637 & 18,7 & 13,39 & +0,341 & 4,69 & +1,19 & 7,51 & 0,401 \\ 30 \ldots \ldots \ldots \ldots & 625 & 9,9 & 9,13 & +0,104 & 4,62 & +0,36 & 4,15 & 0,419 \\ 34 \ldots \ldots \ldots \ldots & 596 & 16,3 & 10,61 & +0,347 & 4,49 & +1,21 & 4,91 & 0,301 \\ 2 \ldots \ldots \ldots \ldots & 616 & 6,3 & 8,03 & +0,312 & 4,59 & +1,09 & 2,35 & 0,372\end{array}$

D. EXPÉRIENCES DE BONNELLES - VACHES A GRAND RENDEMENT

\begin{tabular}{|c|c|c|c|c|c|c|c|c|}
\hline $28 \ldots \ldots \ldots \ldots$ & 650 & 24,8 & 14,62 & $-0,152$ & 4,75 & $-0,53$ & 10,40 & 0,420 \\
\hline 27 & 633 & 24,4 & 14,70 & $-0,048$ & 4,67 & $-0,17$ & 10,20 & 0,418 \\
\hline . & 682 & 21,3 & 13,22 & $+0,658$ & 4,90 & $+2,30$ & 6,02 & 0,283 \\
\hline 20 . & 606 & 20,1 & 12,58 & $-0,400$ & 4,53 & $-1,40$ & 9,45 & 0,469 \\
\hline 12. & 608 & 27,5 & 15,08 & $+0,105$ & 4,54 & 0,37 & 10,17 & 0,370 \\
\hline 16 . & 606 & 25,0 & 14,04 & $-0,096$ & 4,53 & 0,34 & 9,85 & 0,394 \\
\hline
\end{tabular}

Voici de quelle manière ces résultats se groupent autour de leur valeur moyenne, 0 u. 375 :

Limites declasses (unités) de 0,25 de 0,30 de 0,35 de 0,40 de 0,45 de 0,50 à 0,30 à 0,35 à 0,40 à 0,45 à 0,50 à 0,55

Nombre de données par
classe .............
2
3
8
5
2
1

Il existe donc bien, entre un animal et un autre, un important écart entre les rations pratiquement nécessaires pour la production d'un kilogramme de lait. A côté des vaches dont la mamelle travaille d'une façon économique, comme Berne, Leeuwarder, Gérard. 6,32 et 34 , il en est d'autres qui paraissent gaspiller l'énergie utile de leurs aliments, comme Châtaigne, Bonne Mère et 20. Malheureusement, la modicité des crédits dont nous disposions ne nous a pas permis de prolonger comme nous le désirions ces expériences, afin de vérifier si les écarts ainsi constatés persistaient aux différentes époques de l'existence d'une même vache. Il resterait également à rechercher si ces écarts sont transmissibles par hérédité.

En raison de l'importance que pourrait avoir une solution positive de ce problème, il faut souhaiter que les expériences du genre de celles que nous venons de relater puissent se multiplier dans l'avenir, avec la collaboration des Associations de livres généalogiques et de contrôle laitier. 


\title{
BIBLIOGRAPHIE
}

des ouvrages consultés au cours du présent travail.

H. T. Converse. Journal of Dairy Science, V IX, no 4, p. 388. Effets, sur la production laitière, de rations plus fortes que celles indiquées par les normes d'Haecker, d'Eckles et de Savage.

C. H. Eckles et T. W. Gullickson. Journal of Agricultural Research, vol, 42, $\mathrm{n}^{\circ}$, Washington, May 1931, p. 603-616. Besoins nutritifs pour la croissance normale des vaches laitières.

B. Forbes. Journal of Dairy Science, V IX, no 4, p. 373. Besoins d'énergie pour les vaches laitières,

A.-M. Lmroy. Comptes rendus du Congrès de l' Alimentation du Bétail et du Contrốle laitier. Société Nationale d'Encouragement à l'Agriculture. Paris, 1925. Nouvelles étud əs sur la valeur pratique des tables d'alimentation pour vaches laitières.

A.-M. LERoy. Revue de Zootechnie, n० 2-3, février-mars 1933. Conseils aux éleveurs de vaches laitières.

Mergs et Converse. Journal of Dairy Science, V, vol. VIII, n ${ }^{\text {os }} 5$ et 11, p. 177 et 523. Besoins d'énergie des vaches laitières.

Stations de recherches danoises :

Bulletin 136, 1931. Influence du taux de protéine de la ration sur la production laitière.

Bulletin 144, 1932. Recherches sur l'alimentation des vaches laitières avec betteraves fourragères, demi-sucrières et sucrières.

\section{LA PASTEURISATION DU LAIT EN VUE DE LA FABRICATION DES FROMAGES (1)}

\author{
par JEAN PIEN
}

- Docteur ès Sciences, Ingénieur chimiste (I. C. R.)

Directeur des Laboratoires des "Fermiers Réunis"

L'une des grandes préoceupations actuelles des techniciens du lait et des hygiénistes, est la pasteurisation obligatoire du lait.

La preuve est faite des dangers dn lait cru et des bienfaits de la pasteurisation. Tout récemment encore, au XIVe Congrès de Chimie Industrielle (Octobre 1934), nous avons rappelé et développé les raisons pour lesquelles le lait eru est ou peut être un danger, et nous avons également apporté les preuves de l'efficacité de la pasteurisation.

Ces raisons ont tellement paru convaincantes, qu'un vœu tendant à rendre obligatoire la pasteurisation du lait, a été adopté à l'unanimité.

Nous pouvons d'ailleurs dire que la question est à l'étude dans

(1) Extrait du Bulletin de l'Association des Chimistes de Sucrerie, Distillerie et Indrustrie Agricoles (156, boulevard Magenta) ; numéro de décembre 1934. 